\title{
3D Numerical Investigating of Flow Field and Energy Separation in Counter-flow Vortex Tube
}

\author{
Mohammad O. Hamdan ${ }^{1}$, Hashem M. Alargha ${ }^{2}$, Ali Hilal-Alnaqbi ${ }^{2}$, Bobby Mathew ${ }^{2}$ \\ ${ }^{1}$ American University of Sharjah \\ P.O. Box 24444, Mechanical Engineering Department, Sharjah, UAE \\ mhamdan@aus.edu \\ ${ }^{2}$ United Arab Emirates University \\ P.O. Box 15551, Mechanical Engineering Department, Alain, UAE \\ hashem.alargha@gmail.com; alihilal@uaeu.ac.ae; bmathew@uaeu.ac.ae
}

\begin{abstract}
A numerical analysis is carried out in this study in order to understand the flow field and the associated temperature separation in a Ranque-Hilsch vortex tube. A three dimensional computational fluid dynamics model, using ideal gas compressible flow assumptions, is employed to predict the performance of a vortex tube. The standard k-epsilon CFD turbulent model is adopted in this study. The study focuses on an insulated counter flow vortex tube with four tangential inlet streams, one axial hot outlet stream and one axial cold outlet stream. The study shows that one can numerically predict the behaviour of energy separation using ideal gas assumption. The numerical study shows that with an adapted vortex tube size a maximum temperature separation is achieved at optimum pressure value of 4 bar. For insulated tube, as tube length increases, the energy separation increases until it approaches an asymptote value. An optimum diameter exist where energy separation reach maximum value.
\end{abstract}

Keywords: Ranque-Hilsch vortex tube, temperature separation, numerical simulation, CFD, counter-flow vortex tube

\section{Introduction}

The Ranque-Hilsch vortex tube (RHVT) is a thermal mechanical device that passively separates a high pressure gas flow to two lower pressure streams; one of the streams is colder than the inlet flow while the other stream is hotter than the inlet flow. The vortex tube has no moving parts and the energy separation occurs due to swirl flow without requiring any external work or heat transfer. The vortex tube has been discovered by Ranque [1-2] who has encountered the vortex tube phenomenon while experimentally investigating the vortex tube pump in 1928. Later in 1945, Rudolf Hilsch [3] experimentally tested the vortex tube thermal performance under different inlet pressure values and different geometrical parameters.

It has been known that the vortex tube is a low cost effective solution for many cooling problems such as cooling soldered parts, cooling gas samples, electronic component cooling, cooling heat seals, cooling of firemen's suit, cooling of cutting tools [4] and cooling environmental chamber [5,6]. Despite its small cooling capacity, the RHVT is very useful for particular thermal management applications because of its simplicity, compactness, light weight, robustness, reliability, low maintenance cost and safety [7]. The RHVT can be classified into two types [8]: (1) the counter-flow RHVT and (2) the uni-flow RHVT. In the counter flow RHVT type, the cold flow moves in the opposite direction of the hot stream, while in the uni-flow, the hot and cold streams flow in the same direction. In general, the counter-flow RHVT is recommended over the uni-flow RHVT for its efficient energy separation [8].

The separation mechanism inside the vortex tube has not completely understood until today [9]. Recent work by Polihronov and Straatman [10,11] proposed that the temperature separation (rotational cooling) is due to angular propulsion. Vortex tube is covered extensively in literatures through experimental and numerical analysis. The experimental work of Nimbalkar and Muller [12] indicated that there is an optimum diameter of the cold end orifice for achieving maximum energy separation. Also, the results [12] have showed that the maximum value of energy separation is always reachable at a $60 \%$ of the cold mass fraction regardless of the orifice diameter and the inlet pressure. The optimum diameter to the length ratio of the hot side has been investigated by Dincer et al. $[13,14]$. The vortex tube performance has 
been investigated for three different working gases [15]: air, oxygen and nitrogen and the results have been reported using streakline visualizations in a vortex tube made of acrylic. Aydın and Baki [15] indicated that inlet pressure and cold mass fraction are the most important operating parameters.

The effect of nozzle number has been investigated by different scientist [16-20]. Eiamsa-ard [16] reported that the increase in the nozzle number and the supply pressure leads to the rise of the swirl/vortex intensity and thus the energy separation in the tube. Hamdan et al. [17] has showed that vortex tube performance changes dramatically with number of nozzles and that an optimum number provides an optimum performance [18].

Xue and Arjomandi [21] reported that the vortex angle has direct effect on vortex tube and that smaller vortex angles demonstrate a larger temperature difference and superior performance for the heating efficiency of the vortex tube. It was reported [9] that cooling the vortex tube improves the cooling capability by 5-9\%. The effect of vortex entrance condition is reported in [22] which indicated that vortex tube can be improved by rounding the entrance. Scientists worked in improving vortex tube by redesigning the nozzle gas inlet and utilizing diffuser vortex tube which showed reasonable enhancement [23]. A modification to vortex tube has been proposed [24] by introducing a double-circuit vortex tube which showed improvement in the vortex tube performance.

In literature, several studies have been conducted to analyze vortex tube operation using computational fluid dynamics which showed acceptable agreement with published data [25-28]. A full discussion of 3D numerical study is presented in by Behera et al. [26] in which they reported a maximum COP of 0.59 as a heat pump and 0.83 as a refrigerator. Behera et al. [26] has reported numerically that the secondary flow degrade the performance of the vortex tube. It has been reported numerically that the magnitude of velocity angle is reversely proportional to the vortex pipe diameter [28].

\section{Numerical Formulation}

The numerical study is performed using ANSYS Fluent which is a commercial computation fluid dynamics tool. The detailed dimensions, boundary conditions and mesh independent study are presented in next sections. The study addresses the effect of inlet pressure, the mass fraction, the tube length and diameter on the performance of the selected vortex tube geometry. Hence the study is divided to four parts. The first part of the study focuses on the effect of changing the inlet pressure while fixing all other conditions such as inlet temperature and outlet pressure. Multiple values for inlet pressure are tested ranging between 3 and 6 bar while having 0.7 bar fixed at the hot outlet and zero gauge pressure at the cold exit with a $300 \mathrm{~K}$ inlet temperature. Second, the study focuses on evaluating the effect of mass fraction where energy separation are tested under wide range of mass fraction and under the optimum pressure that is determined from the first part. Thirdly the effect of vortex tube on energy separation is investigated and finally the effect of tube diameter.

\subsection{Geometry and dimensions the of model}

As illustrated in Figure (1), the vortex tube used on this study has a working length of $97 \mathrm{~mm}, 4$ inlet nozzles of 1.6 $\mathrm{mm}$ diameter, cold outlet diameter of $5.43 \mathrm{~mm}$ and a diameter of $14.48 \mathrm{~mm}$. The selected dimensions are based on actual operating vortex tube size. Figure (2) shows the full three-dimensional geometry of the vortex tube and the controllable conical valve at the hot outlet. As illustrated, the vortex tube does not have any moving parts, just compressed air enters simply through the four tangential inlet nozzles then a radial temperature gradient takes place due to the rotating flow.

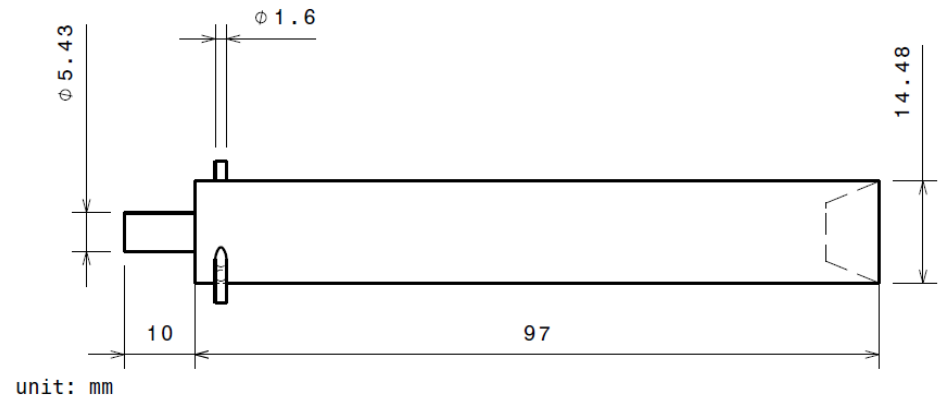

Fig. 1: Side view of a counter flow vortex tube with four tangential inlets vortex tube (only two nozzles are shown in the side view). 


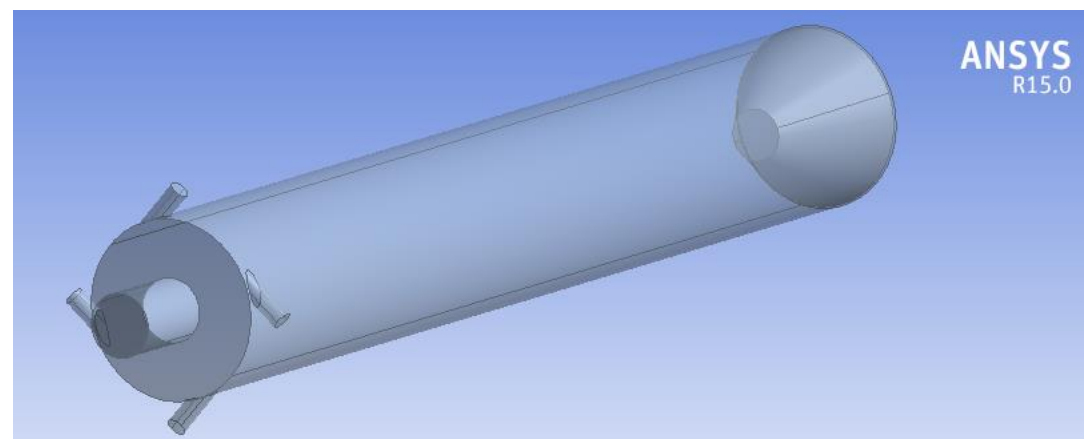

Fig. 2: 3D vortex tube geometry.

\subsection{Boundary conditions}

Multiple values for inlet pressure are tested ranging between 3 and 6 bar while having 0.7 bar fixed at the hot outlet and zero gauge pressure at the cold exit with a $300 \mathrm{~K}$ inlet temperature. No slip conditions were applied at interior walls and air is considered as an ideal gas while the walls are kept under adiabatic condition. Since vortex tube flow is always highly turbulent, the standard k- $\varepsilon$ model has been adopted in this study.

\subsection{Mesh independent study}

Mesh independence study was conducted to assure that the final solution is independent of the mesh size. The independence of the results is determined based on observing the change of the hot outlet temperature. It is clear from Figure (3) that there is no further significance variation in the results driven by increasing the number of nodes beyond 1.7 million nodes, hence mesh size of 1.7 million nodes has been chosen as the optimum mesh size. The mesh shown in figure (4), contains 1.7 million nodes. An inflation layer near the wall is used with a total thickness of 1 mm divided into 20 layers with growth rate of 1.2.

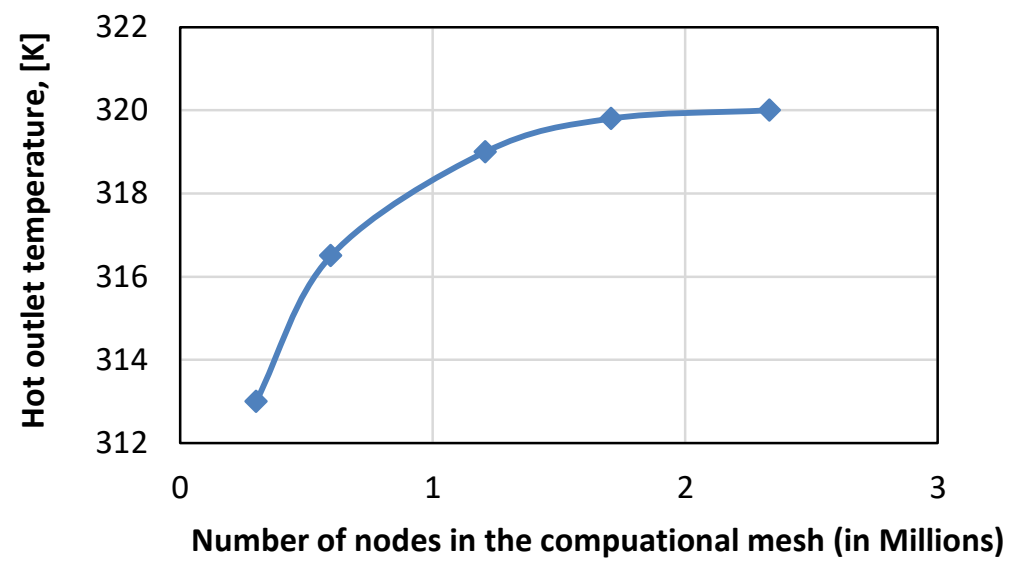

Fig. 3: Evaluating mesh independency by monitoring the hot outlet temperature versus number of nodes.

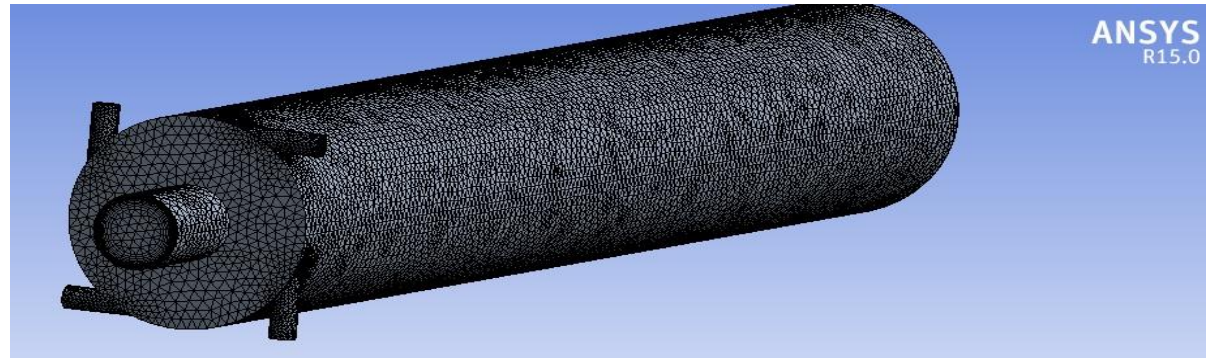

Fig. 4: vortex tube mesh. 


\section{Results and discussion}

\subsection{Changing inlet pressure}

The temperature difference between both hot and cold outlet is plotted in figure (5) while fixing all other conditions. The maximum temperature difference has been found to be $42.3 \mathrm{~K}$ at 4 bars inlet pressure. As pressure increases separation increases however after specific value ( 4 bar for current design), the mixing between the two outlet stream become dominant which deteriorate the energy separation.

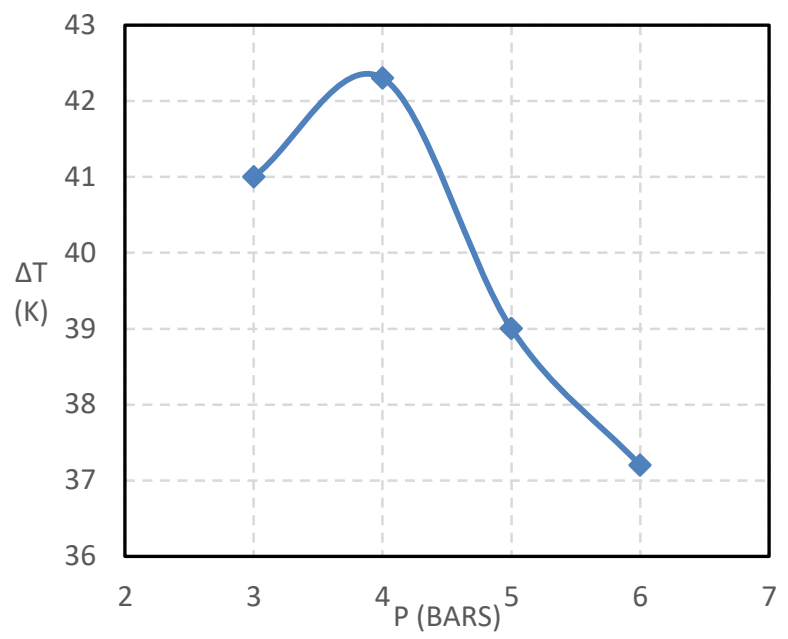

Fig. 5: Temperature difference (K) versus inlet pressure (bar).

The temperature separation is clearly shown in figures (6) for the 3 bar case. The figure which show a $2 \mathrm{D}$ view of the temperature contours. It shows that the $300 \mathrm{~K}$ stream splits into two different streams; one is hotter than the inlet itself while the other is colder. With the hot outlet pressure fixed at 0.7 bars, it is found that a maximum temperature difference of about 42 degrees takes a place to split the inlet stream to a hotter $310.3 \mathrm{~K}$ stream and a $268 \mathrm{~K}$ cold stream. Figure (7) shows a 3D view of separation. Figure (7) shows three dimensional stream lines of the flow. The outer shell represents the free vortex while the core stream line stands for the forced vortex. The flow is highly turbulent along the tube.

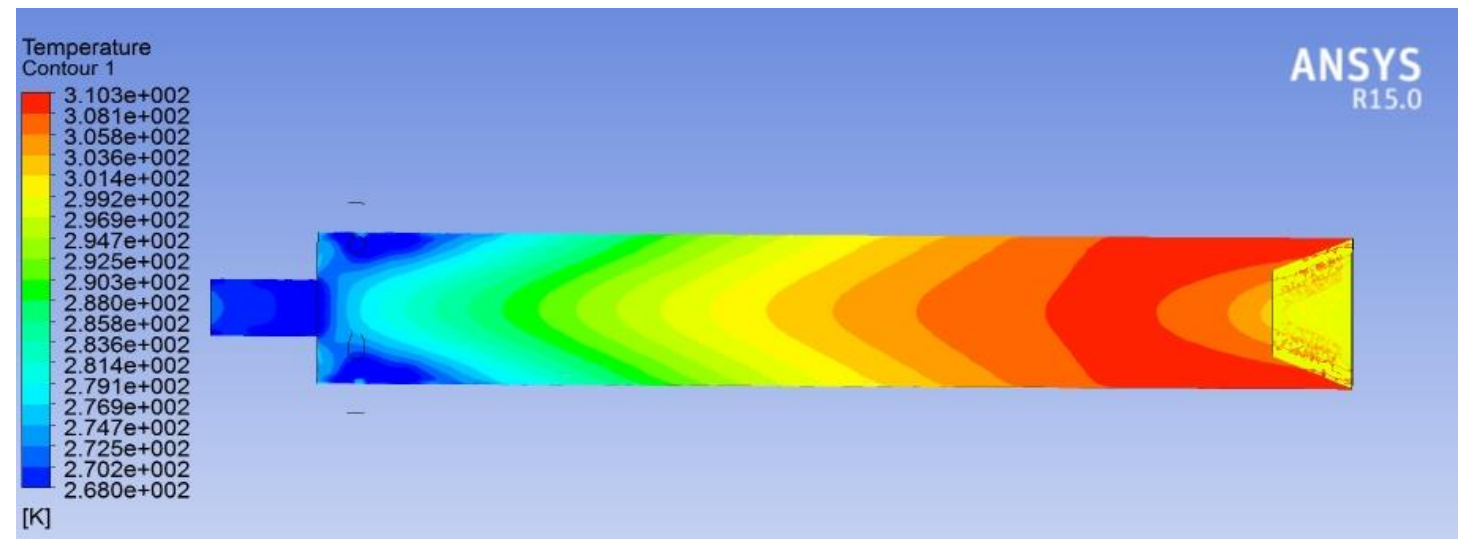

Fig. 6: Temperature separation at 4 bar inlet pressure (2D view). 


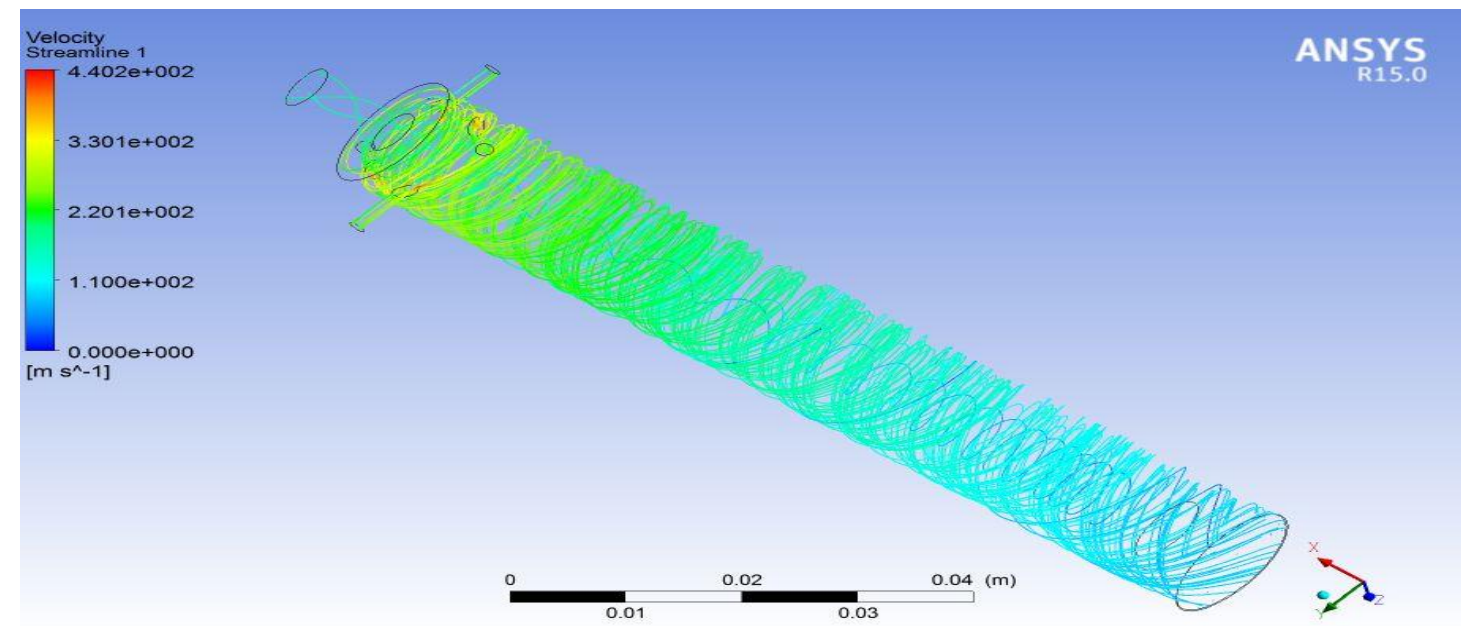

Fig. 7: velocity stream lines for 4 bar inlet pressure (3D view).

\subsection{Changing cold mass fraction}

Figure (8) illustrates the variation on temperature difference as the hot outlet mass fraction flow rate changes while fixing the inlet pressure at 4 bar. The hot outlet mass fraction flow is changed from 0.2 up to 0.8 by changing the outlet pressure at the hot side. As shown, the temperature difference decreases as the hot outlet mass fraction increases which means flow separation is getting weaker hence energy separation is getting deteriorated.

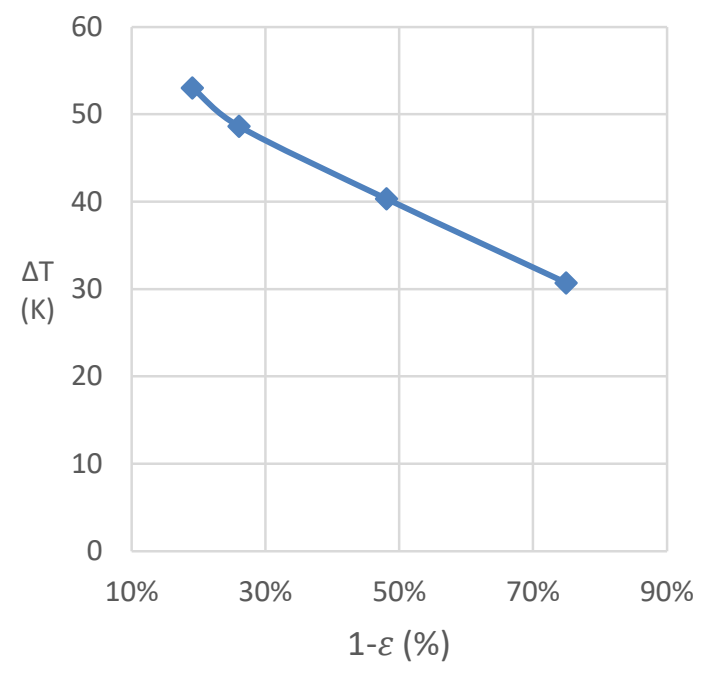

Fig. 8: hot outlet temperature vs hot outlet mass flow rate.

\subsection{Changing length}

Changing the length consisted of changing the total length $\mathrm{L}$ of the vortex tube from $60 \mathrm{~mm}$ up to $800 \mathrm{~mm}$. This was done to test the optimum point at which the vortex tube will operate. As shown in Figure (9), the temperature different increases as tube length increase and keep increasing until reaching asymptote value. This is expected since as tube get longer the vortex is properly formed and after that any extra length will not help in achiving any additional enhancement in energy separation. In experimental testing, one found that energy separation increases with longer tube however after specific length the performance deteriorate which happen due to heat loss. In the CFD analysis the wall has been assumed insulated which caused the monotonic trend shown in figure (9). 


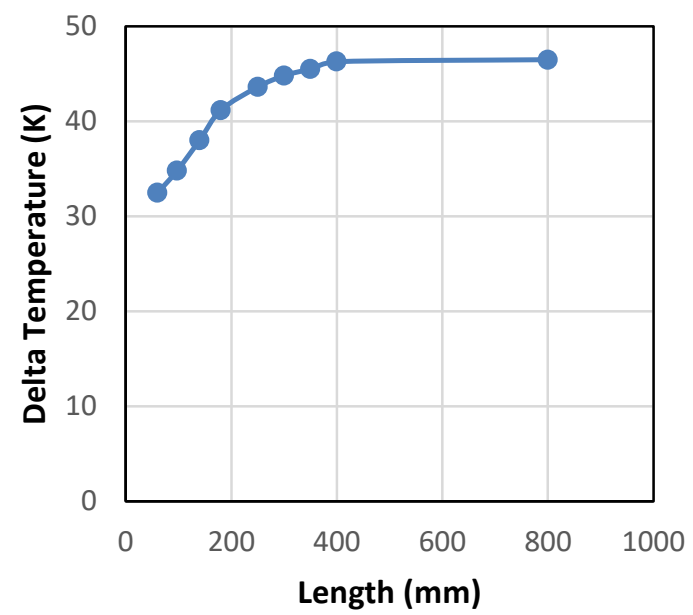

Fig. 9: Change in temperature versus the Vortex tube length.

\subsection{Changing Tube Diameter}

Five simulations were done using five different diameters for the vortex tube as shown in figure (10). The optimum diameter for a maximum temperature difference has been found to be $10 \mathrm{~mm}$. This gives a temperature difference of 39.6 degrees Celsius. The reason as of having an optimum value is the two conflicting effect that the diameter has on energy separation. As diameter increases, less friction occur causing less resistance and boosting energy separation however as diameter grow to a specific value near optimum diameter the trend gets reversed. Once the diameter exceeds such optimum diameter, the effect of weaker vortex causes the energy separation to deteriorate. The vortex get weaker is explained by conservation of mass which mean as area increases the average velocity decreases.

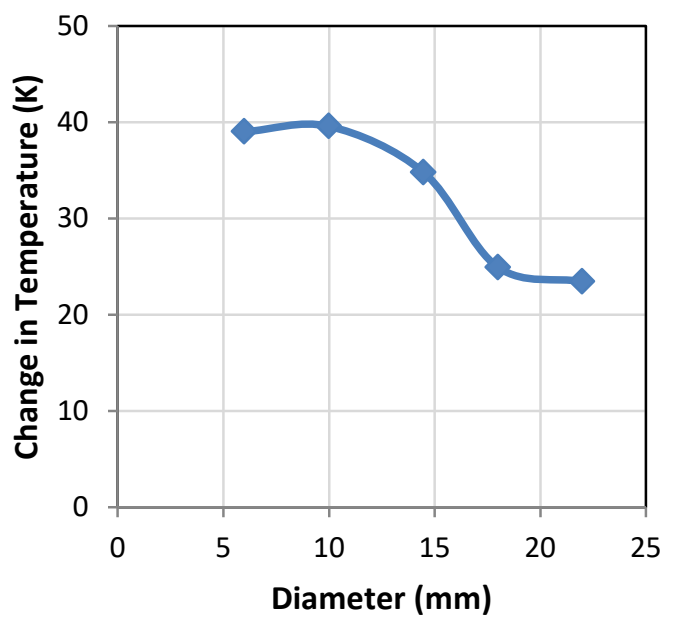

Fig. 10: Change in temperature versus the Vortex tube diameter.

\section{Conclusion}

In conclusion, numerical examination is being done to explore and simulate a 3D vortex tube using CFD software Fluent Ansys. CFD simulation used to improve the efficiency of the vortex tube. It helps to examine different parameters in case to improve the design. Also, it provides a safety environment, save time and money. The analysis yielded the following:

Four bar inlet pressure gives the maximum temperature separation. Moreover, the utilized mesh should be fine and achieved properly to have results with minimum error and clear energy separation. Our results show that 4 bars inlet 
pressure gives the maximum temperature difference. The hot outlet temperature decreases as the hot fraction increase. Finally the temperature differences decrease as the hot fraction increases.

\section{Acknowledgements}

This research was supported by [UAE University, 31R071-Research Center- ECEER -5-2015]. We thank our colleagues from UAE University who provided insight and expertise that greatly assisted the research. We thank our student Youssef ElKassem and Braa Kakah for assistance and support in running/testing different CFD codes.

\section{References}

[1] G. Ranque, "Experiments on expansion in a vortex with simultaneous exhaust of hot air and cold air," $J$ Phys Radium, Paris, vol. 4, pp. 112-114, 1933.

[2] G. Ranque, "Method and apparatus for obtaining from a fluid under pressure two outputs of fluid at different temperatures," US patent, 1:952, 281, 1934.

[3] R. Hilsch, "The use of the expansion of gases in a centrifugal field as cooling process," Rev. Sci. Instrum, vol. 18, no. 2, pp. 108-113, 1947.

[4] B. Alsayyed, M. O. Hamdan, S. Aldajah, "Vortex Tube Impact on Cooling Milling Machining," ASME 2012 International Mechanical Engineering Congress and Exposition, pp. 773-776.

[5] T. J. Bruno, "Laboratory Applications of the Vortex Tube," J. Chem. Educ., 1987, vol. 64, no. 11, pp. 987.

[6] M. O. Hamdan, A. Alawar, E. Elnajjar, W. Siddique, "Feasibility of Vortex Tube Air-Conditioning System," ASME/JSME 2011 8th Thermal Engineering Joint Conference, T20004-T20004-5.

[7] J. Lewins, A. Bejan, "Vortex tube optimization theory," Energy, vol. 24, pp. 931-43, 1999.

[8] S. Eiamsa-ard, K. Wongcharee, P. Promvonge, "Experimental investigation on energy separation in a counter-flow Ranque-Hilsch vortex tube: Effect of cooling a hot tube," International Communications in Heat and Mass Transfer, vol. 37, pp. 156-162, 2010.

[9] S. Eiamsa-ard, P. Promvonge, "Review of Ranque-Hilsch effects in vortex tubes," Renewable and Sustainable Energy Reviews, vol. 12, pp. 1822-1842, 2008.

[10] J. Polihronov, A. Straatman, "Thermodynamics of angular propulsion in fluids," Physical Review Letters 054504, 2012.

[11] J. Polihronov, A. Straatman, "The vortex tube effect without walls," Canadian Journal of Physics, DOI: 10.1139/cjp-2014-0227, 2015.

[12] S. U. Nimbalkar, M. R. Muller, "An experimental investigation of the optimum geometry for the cold end orifice of a vortex tube," Applied Thermal Engineering, vol. 29, pp. 509-514, 2009.

[13] K. Dincer, S. Baskaya, B. Z. Uysal, I. Ucgul, "Experimental investigation of the performance of a Ranque-Hilsch vortex tube with regard to a plug located at the hot outlet," International Journal of Refrigeration, vol. 32, pp. 87-94, 2009.

[14] K. Dincer, S. Tasdemir, S. Baskaya, B. Z. Uysal, "Modeling of the effects of length to diameter ratio and nozzle number on the performance of counterflow Ranque-Hilsch vortex tubes using artificial neural networks," Applied Thermal Engineering, vol. 28, pp. 2380-2390, 2008.

[15] O. Aydın, M. Baki, "An experimental study on the design parameters of a counterflow vortex tube," Energ, vol. 31, pp. 2763-2772, 2006.

[16] S. Eiamsa-ard, "Experimental investigation of energy separation in a counter flow Ranque-Hilsch vortex tube with multiple inlet snail entries," International communications in Heat and Mass Transfer, vol. 37, no. 6, pp. 637-643, 2010.

[17] M. O. Hamdan, A. Alawar, E. Elnajjar, W. Siddique, "Experimental Analysis on Vortex Tube Energy Separation Performance," Journal of Heat and Mass Transfer, DOI: 10.1007/s00231-011-0824-6, 2011.

[18] M. O. Hamdan, B. Alsayyed, E. Elnajjar, "Nozzle parameters affecting vortex tube energy separation performance," Heat and Mass Transfer, vol. 49, no. 4, pp. 533-54.

[19] V. Kirmaci, O. Uluer, "An experimental investigation of the cold mass fraction, nozzle number and inlet pressure effects on performance of counter flow vortex tube," ASME J. Heat. Transfer, vol. 131, pp. 081701-081709, 2009. 
[20] V. Kirmaci, O. Uluer, "The Effects of Orifice Nozzle Number on Heating and Cooling Performance of Vortex Tubes: An Experimental Study," Instrumentation Science \& Technology, vol. 36, no. 5, pp. 493-502, DOI: 10.1080/10739140802234923, 2008.

[21] Y. Xue, M. Arjomandi, "The effect of vortex angle on the efficiency of the Ranque- Hilsch vortex tube," Experimental Thermal and Fluid Science, vol. 33, pp. 54-57, 2008.

[22] C. M. Gao, K. J. Bosschaart, J. C. H. Zeegers, A.T. A. M. de Waele, "Experimental study on a simple RanqueHilsch vortex tube," Cryogenics, vol. 45, pp. 173-183, 2005.

[23] Y. T. Wu, Y. Ding, Y. B. Ji, C. F. Ma, M. C. Ge, "Modification and experimental research on vortex tube," International Journal of Refrigeration, vol. 30, pp. 1042-1049, 2007.

[24] S. A. Piralishvili, V. M. Polyaev, "Flow and Thermodynamic Characteristics of Energy Separation in a DoubleCircuit Vortex Tube An Experimental Investigation," Experimental Thermal and Fluid Science, vol. 12, pp. 399410, 1996.

[25] U. Behera, P. J. Paul, K. Dinesh, S. Jacob, "Numerical investigations on flow behavior and energy separation in Ranque-Hilsch vortex tube," Int J Heat Mass Transf., vol. 51, no. 25-26, pp. 6077-6089, 2008.

[26] U. Behera, P. J. Paul, S. Kasthurirengan, R. Karunanithi, S. N. Ram, K. Dinesh, S. Jacob, "CFD analysis and experimental investigations towards optimizing the parameters of Ranque-Hilsch vortex tube," Int $J$ Heat Mass Transf., vol. 48, pp. 1961-1973, 2005.

[27] S. Eiamsa-ard, P. Promvonge, "Numerical investigation of the thermal separation in a Ranque-Hilsch vortex tube," Int J Heat Mass Transf, , vol. 50, pp. 821-832, 2007.

[28] N. F. Aljuwayhel, G. F. Nellis, S. A. Klein, "Parametric and internal study of the vortex tube using a CFD model," Int J Refrig., vol. 28, pp. 442-450, 2005. 